\title{
Diseño, construcción y control de una plataforma Bola-Plano
}

\author{
I. Oliva-Leal ${ }^{\mathrm{a}, *}$, O. Hernández-Durán ${ }^{\mathrm{a}}$, E. A. Gómez-Vidal ${ }^{\mathrm{a}}$, J. O. Trejo-Salgado ${ }^{\mathrm{b}}$, R. Villafuerte-Segura ${ }^{\mathrm{a}}$, C. Cuvas-Casillo ${ }^{\mathrm{a}}$, J. P. \\ Ordaz-Oliver ${ }^{\mathrm{a}}$ \\ ${ }^{a}$ Instituto de Ciencias Básicas e Ingeniería, Universidad Autónoma del Estado de Hidalgo, Hidalgo, México. \\ ${ }^{b}$ Facultad de Ingeniería, Universidad Tecnológica de Tepeji, Hidalgo, México.
}

\begin{abstract}
Resumen
En este documento se presenta el diseño, construcción y control de una plataforma bola-plano. Todos los componentes de la plataforma fueron diseñados con el programa de Diseño Asistido por Computadora (CAD) llamado SolidWork e impresos con la ayuda de una impresora 3D. Además, a partir del modelo matemático del sistema, se sintoniza una ley de control ProporcionalDerivativa (PD), con el objetivo de estabilizar la bola en la plataforma. La ley de control fue implementada en el software LabVIEW empleando la tarjeta de adquisición de datos MyRIO-1900. También, se evidencian mediante gráficas los resultados experimentales obtenidos.
\end{abstract}

Palabras Clave: Plataforma bola-plano, Diseño Asistido por Computadora, controlador PD, estabilización de sistemas.

\section{Introducción}

La plataforma bola-plano (ball on plane) es una extensión de la plataforma conocida como bola-viga (ball on beam). La plataforma bola-viga es un sistema de un grado de libertad cuyo propósito es medir y controlar el desplazamientos de una bola sobre una viga, (Keshmiri et al., 2012). Mientras que la plataforma bola-plano es un sistema de dos grados de libertar que consiste en medir y controlar el desplazamientos de una bola sobre un plano, (Wang et al., 2009; B. A. López, 2018).

La plataforma bola-viga ha sido estudiada durante varias décadas como un sistema no lineal clásico. Algunos autores han realizado aportaciones sustanciales en el diseño y control de este tipo de plataformas, entre los cuales se pueden mencionar los siguientes: (Hauser et al., 1992), donde el autor aplica un control no lineal mediante la aproximación entrada-salida, en ese mismo año en (Benbrahim et al., 1992) se muestra la aplicación del aprendizaje en tiempo real para controlar la posición de la bola sobre una viga, más adelante se utilizan redes neuronales para controlar este tipo de sistemas (Tack et al., 1999). También, se ha usado el método indirecto de Lyapunov en (Garzón Melo et al., 2016).

\footnotetext{
*Autor en correspondencia.

Correos electrónicos: idae1921013@gmail.com (I. Oliva-Leal ), ozielhd@gmail. com (O. Hernández-Durán), emmanuel.gomezv@outlook.com (E. A. Gómez-Vidal), olimpots@hotmail.com (J. O. Trejo-Salgado), villafuerte@uaeh.edu.mx (R. Villafuerte-Segura), carlos_cuvas@uaeh.edu.mx (C. Cuvas-Casillo), jesus_ordaz@uaeh.edu.mx (J. P. Ordaz-Oliver)
}

En la plataforma bola-plano se pueden aplicar diversos tipos de leyes de control, en (Awtar et al., 2002) se implementa un controlador Proporcional-Integral-Derivativo PID, a pesar de ser un control lineal se obtienen buenos resultados y su aplicación es muy sencilla, otra opción, es la aplicación de controles no lineales, como es el caso de los controles por modos deslizantes utilizado en (Liu and Liang, 2010), resultando una buena opción, dado que la planta se vuelve robusta ante perturbaciones, además se logra convergencia en tiempo finito. Las redes neuronales difusas fueron aplicadas en (Dong et al., 2011), las cuales ayudan a mejorar la sintonización de un controlador PID. En trabajos más recientes se aplican algoritmos mejorados, tal es el caso del I+PD utilizado en (Mochizuki and Ichihara, 2013), que tiene la particularidad de mejorar la respuesta a los cambios en la referencia.

En este manuscrito se aborda aspectos importantes para el diseño, construcción y control de una plataforma bola-plano, con el propósito de brindar herramientas que coadyuven en el proceso enseñanza-aprendizaje de las ciencias duras (básicas) y sus aplicaciones. El diseño y construcción fue resuelto utilizando el software SolidWork y la tecnología de impresión 3D, respectivamente. El problema de control fue resulto mediante la implementación de un PD, cuya sintonización fue vía asignación de polos. Mientras que su aplicación fue usando el software LabVIEW y una tarjeta de adquisición de datos MyRIO1900. Comúnmente, para medir la posición de la bola en el plano se utilizan cámaras y software de visión, en este proyecto se utiliza una pantalla táctil resistiva, la cual será nuestro plano, con el objetivo de disminuir el costo final de la plataforma y me- 
dir de manera directa y eficiente la posición de la bola. Además, para realizar la lectura de la pantalla táctil se utilizó el controlador STMPE610, el cual solo cuanto con librerías para Arduino y Raspberry, por lo cual fue necesario la creación total de una librería para LabVIEW.

El presente documento está estructurado de la forma siguiente: en la Sección 2 se muestran los resultados preliminares tales como, la descripción del modelo matemático utilizado para representar al sistema, el diseño y la construcción de la plataforma experimental considerando la estructura mecánica y electrónica, además se explica la adquisición de datos mediante el software LabVIEW y el controlador STMPE610 para obtener la posición de la bola en el plano (pantalla). La sintonización del control PD para la estabilización de la plataforma bola-plano se presenta en la Sección 3, mientras que los resultados experimentales del sistema en lazo cerrado se muestran en la Sección 4. Finalmente el trabajo termina con las conclusiones en la Sección 5.

\section{Resultados Preliminares}

En esta sección se describe la obtención del modelo matemático, el diseño y construcción de la plataforma bola-plano.

\subsection{Modelado matemático del bola-plano}

En la Figura 1 se muestra un esquema general de la plataforma conocida como bola-plano. Básicamente, esta plataforma se compone de tres partes principales. a) una pantalla táctil resistiva empleada como plano de la plataforma $(X, Y)$ y como sensor para medir la posición de la bola, b) dos servomotores con bielas para variar la posición angulas $\theta_{X}$ y $\theta_{Y}$ de la pantalla táctil y c) un eje conectado al centro de la pantalla que funciona como pivote cuando se varían las posiciones angulares.
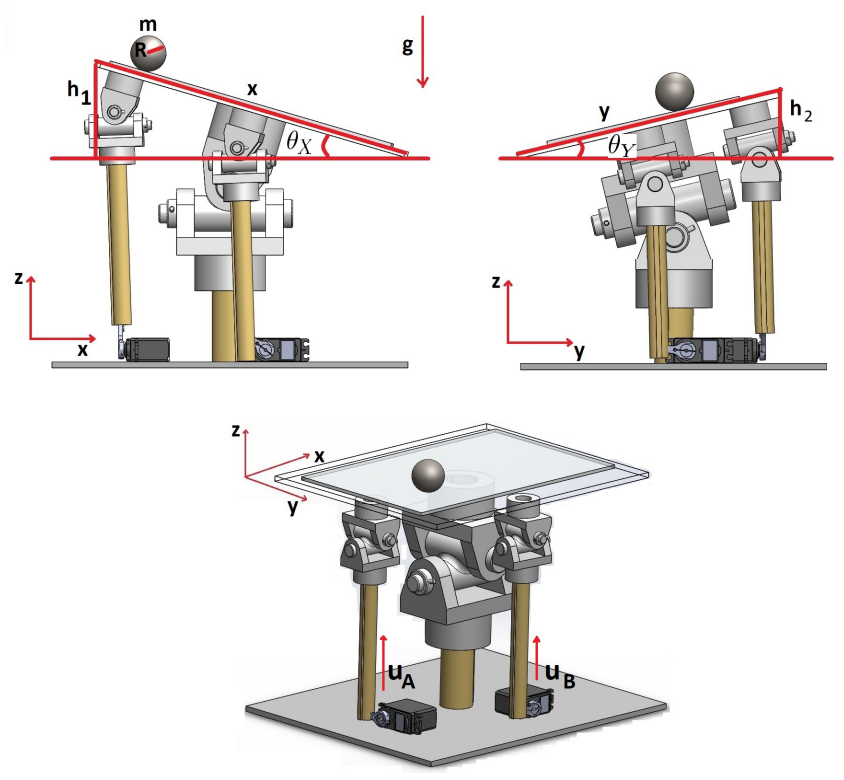

Figura 1: Definición de los ejes coordenados y ángulos de giro.

\begin{tabular}{|l|l|}
\hline Var. & Descripción \\
\hline \hline$X$ & desplazamiento (m) de la bola sobre el eje $X$ \\
\hline$Y$ & desplazamiento (m) de la bola sobre el eje $Y$ \\
\hline$\theta_{X}$ & ángulo (rad) entre el eje $X$ y el plano horizontal \\
\hline$\theta_{Y}$ & ángulo (rad) entre el eje $Y$ y el plano horizontal \\
\hline
\end{tabular}

Tabla 1: Parámetros y variables físicas del bola-plano.

En la figura anterior, cada variable representa un parámetro que se describe en la Tabla 2.1.

Como es bien conocido, para describir el comportamiento de un sistema dinámico en términos del trabajo y la energía almacenados en la plataforma bola-plano se utiliza la formulación de Euler-Lagrange como se muestra a continuación (Mancera, 2016).

$$
\frac{d}{d t}\left(\frac{\partial L}{\partial \dot{q}_{j}}\right)-\left(\frac{\partial L}{\partial q_{j}}\right)=Q_{j},
$$

donde $j=1,2,3,4$, y para el presente caso de estudio, $q_{1}=x$, $q_{2}=y, q_{3}=\theta_{x}, q_{4}=\theta_{y}$. Además, $L$ es el Lagrangiano, el cual está definido como la diferencia entre las energías cinética $T$ y potencial $U$ del bola-plano,

$$
L=T-U .
$$

Para el análisis de energías se omite la fricción entre el plano y la bola. La energía cinética de un sistema puede ser representada como la suma de la energía cinética rotacional y energía cinética traslacional para estos dos tipos de movimiento (Colmenares et al., 2012), como se describe en la ecuación siguiente

$$
T=T_{\text {rot }_{1}}+T_{\text {tran }_{1}}+T_{\text {rot }_{2}}+T_{\text {tran }_{2}},
$$

donde $T_{r o t_{1}}+T_{t r a n_{1}}$ representa el movimiento de rodamiento suave de cuerpo rígido uniforme y $T_{\text {rot }_{2}}+T_{\text {tran }_{2}}$ representa la partícula en movimiento que sigue una trayectoria circular los cuales son movimientos que experimenta la bola al rodar. Dichas energías están definidas como se muestra a continuación:

$$
\begin{array}{ll}
T_{\text {rot }_{1}}=\frac{1}{2} I_{e} w_{b}^{2}, & T_{\text {tran }_{1}}=\frac{1}{2} m \dot{r}^{2}, \\
T_{\text {rot }_{2}}=\frac{1}{2} I_{e} \dot{\theta}^{2}, & T_{\text {tran }_{2}}=\frac{1}{2} m \dot{s}^{2},
\end{array}
$$

donde $I_{e}=\frac{2}{5} m R^{2}$ es el momento de inercia de una esfera sólida, $R$ es el radio de la bola, $m$ es la masa de la bola, $w_{b}=r / R$ es la velocidad angular de la bola, $\dot{r}$ es la velocidad lineal de la bola por lo que $\dot{r}^{2}=\dot{x}^{2}+\dot{y}^{2}$, y $\dot{s}$ es la velocidad lineal debido a la rotación, la cual cumple $\dot{s}^{2}=\left(x \dot{\theta}_{x}+y \dot{\theta}_{y}\right)^{2}$.

La energía potencial de la plataforma tomando como referencia el plano puede ser expresada como

$$
U=-m g h,
$$

donde $h=h_{1}+h_{2}$ es la altura de la bola, $\operatorname{con} h_{1}=x \operatorname{sen} \theta_{x}, h_{2}=$ $y \operatorname{sen} \theta_{y}$ y $g=9.81$ es la gravedad. Sustituyendo las ecuaciones (3) y (4) en (2), se obtiene que

$$
L=\frac{1}{2} I_{e}\left[\dot{\theta}^{2}+\left(\frac{\dot{r}}{R}\right)^{2}\right]+\frac{1}{2} m\left[\dot{r}^{2}+\dot{s}^{2}\right]+m g h .
$$


La plataforma al tener dos grados de libertad, uno sobre el ejes $X$ y otro en $Y$, la ecuación anterior se reescribe como

$$
\begin{aligned}
L= & \frac{1}{2} I_{e}\left[\dot{\theta}_{x}^{2}+\dot{\theta}_{y}^{2}+\frac{\dot{x}}{R^{2}}+\frac{\dot{y}}{R^{2}}\right] \\
& +\frac{1}{2} m\left[\dot{x}^{2}+\dot{y}^{2}+\left(x \dot{\theta}_{x}+y \dot{\theta}_{y}\right)^{2}\right] \\
& +m g\left(x \operatorname{sen} \theta_{x}+y \operatorname{sen} \theta_{y}\right) .
\end{aligned}
$$

Aplicando las operaciones formuladas en (1) sobre (5), se obtiene el comportamiento dinámico del bola-plano

$$
\begin{aligned}
\ddot{x} & =b\left[x \dot{\theta}_{x}^{2}+y \dot{\theta}_{x} \dot{\theta}_{y}+g \operatorname{sen} \theta_{x}\right], \\
\ddot{y} & =b\left[y \dot{\theta}_{y}^{2}+x \dot{\theta}_{x} \dot{\theta}_{y}+g \operatorname{sen} \theta_{y}\right], \\
\ddot{\theta}_{x} & =c\left[u_{A}-m\left(2 x \dot{x} \dot{\theta}_{x}+\dot{x} y \dot{\theta}_{y}+x \dot{y} \dot{\theta}_{y}+x y \ddot{\theta}_{y}-g x \cos \theta_{x}\right)\right], \\
\ddot{\theta}_{y} & =d\left[u_{B}-m\left(2 y \dot{y} \dot{\theta}_{y}+\dot{x} y \dot{\theta}_{x}+x \dot{y} \dot{\theta}_{x}+x y \ddot{\theta}_{x}-g y \cos \theta_{y}\right)\right],
\end{aligned}
$$

donde $b=\frac{m}{\frac{I_{e}}{R^{2}}+m}, c=\frac{1}{I_{e}+m x^{2}} \mathrm{y} d=\frac{1}{I_{e}+m y^{2}}$. Ahora, para fines de simplificación, se realizan una serie de suposiciones para obtener una representación dinámica de la plataforma bola-plano. Observe que

$$
b=\frac{m}{\frac{I_{e}}{R^{2}}+m} \frac{m}{\frac{\frac{2}{5} m R^{2}}{R^{2}}+m}=\frac{5}{7},
$$

por lo que, para este caso de estudio, la masa y el radio de la bola no influyen en el modelo matemático. Además, como se vera a continuación, las variable $c$ y $d$ no son consideradas al simplificar el modelo.

En la plataforma bola-plano se presenta un acoplamiento, es decir, la velocidad lineal de la bola en el eje $Y$ depende de la velocidad angular del plato sobre el eje $X$ y viceversa, por lo tanto, para eliminar ese acoplamiento se asumen dos suposiciones, a) la bola no patina, es decir, siempre está rodando, y b) la bola siempre está en contacto con la superficie. Bajo estas suposiciones, el desacoplamiento permite eliminar los términos no lineales $y \dot{\theta}_{x} \dot{\theta}_{y}$ y $x \dot{\theta}_{x} \dot{\theta}_{y}$, además, las aceleraciones angulares del plano son muy cercanas a cero, por lo que se puede considerar que $\ddot{\theta}_{x}=\ddot{\theta}_{y}=0$. Así, realizando el cambio de variable

$$
\begin{array}{llll}
x_{1}=x, & x_{2}=\dot{x}, & x_{3}=\theta_{x}, & x_{4}=\dot{\theta}_{x} \\
x_{5}=y, & x_{6}=\dot{y}, & x_{7}=\theta_{y}, & x_{8}=\dot{\theta}_{y},
\end{array}
$$

el sistema representado en (6) se reduce al espacio de estado dado por

$$
\left[\begin{array}{c}
\dot{x_{1}} \\
\dot{x_{2}} \\
\dot{x_{3}} \\
\dot{x_{4}} \\
\dot{x_{5}} \\
\dot{x_{6}} \\
\dot{x_{7}} \\
\dot{x_{8}}
\end{array}\right]=\left[\begin{array}{c}
x_{2} \\
b\left(x_{1} x_{4}^{2}+g \operatorname{sen} x_{3}\right) \\
x_{4} \\
0 \\
x_{6} \\
b\left(x_{5} x_{8}^{2}+g \operatorname{sen} x_{7}\right) \\
x_{8} \\
0
\end{array}\right]+\left[\begin{array}{ll}
0 & 0 \\
0 & 0 \\
0 & 0 \\
1 & 0 \\
0 & 0 \\
0 & 0 \\
0 & 0 \\
0 & 1
\end{array}\right]\left[\begin{array}{l}
u_{A} \\
u_{B}
\end{array}\right] .
$$

Haciendo una aproximación lineal de (7) se puede llegar a los siguientes modelos a los modelos siguientes para cada uno de los ejes, ver (Mancera, 2016) a) para el eje $X$

$$
\left[\begin{array}{l}
\dot{x}_{1} \\
\dot{x}_{2}
\end{array}\right]=\left[\begin{array}{ll}
0 & 1 \\
0 & 0
\end{array}\right]\left[\begin{array}{l}
x_{1} \\
x_{2}
\end{array}\right]+\left[\begin{array}{c}
0 \\
b g
\end{array}\right] u_{X}, \quad y=\left[\begin{array}{ll}
1 & 0
\end{array}\right]\left[\begin{array}{l}
x_{1} \\
x_{2}
\end{array}\right] .
$$

b) para el eje Y

$$
\left[\begin{array}{l}
\dot{x}_{1} \\
\dot{x}_{2}
\end{array}\right]=\left[\begin{array}{ll}
0 & 1 \\
0 & 0
\end{array}\right]\left[\begin{array}{l}
x_{1} \\
x_{2}
\end{array}\right]+\left[\begin{array}{c}
0 \\
b g
\end{array}\right] u_{y}, \quad y=\left[\begin{array}{ll}
1 & 0
\end{array}\right]\left[\begin{array}{l}
x_{1} \\
x_{2}
\end{array}\right] .
$$

Se puede observar que el modelo matemático del eje $X$ y el eje $Y$ son iguales, salvo $u_{A}$ y $u_{B}$. Definiendo $u=u_{A}=u_{B}$, (8) y (9) tienen la representación en espacio de estado siguiente

$$
\begin{aligned}
& \dot{x}=A x+B u, \\
& y=C x,
\end{aligned}
$$

donde $A \in \mathbb{R}^{2 \times 2}, B \in \mathbb{R}^{2}, C \in \mathbb{R}^{1 \times 2}$, son matrices reales y constantes, $x \in \mathbb{R}^{2}$ es el vector de estado; y $u \in \mathbb{R}, y \in \mathbb{R}$ son la entrada y la salida del sistema, respectivamente. Así, el sistema (6) puede simplificarse a un sistema de la forma (10).

A continuación, se describe el proceso de diseño y construcción de las piezas que componen la plataforma bola-plano mostrada en el Figura 1.

\subsection{Diseño y construcción de la plataforma bola-plano}

El diseño de los componentes de la plataforma fue realizados mediante el software CAD SolidWorks. El maquinado de los componentes fue mediante el empleo de una impresora 3D convencional.

Se diseñaron dos bases, una para el marco y soporte de la pantalla táctil (plano) y otra como base inferior de la plataforma total. Estas dos base se interconectan mediante el mecanismo de giro central (pivote), ver Figura 2. El pivote se estructura de cinco componentes para garantizar un giro libre de la pantalla cuando se varían las posiciones angulares $\theta_{X}$ y $\theta_{Y}$. Se requiere que las posiciones angulares de la pantalla son directamente proporcionales al ángulo de giro de los servomotores, por lo cual, la plataforma tiene una unión fija a su centro, para que la actuación de cada servomotor sea independiente (afecte lo menos posible) del otro.

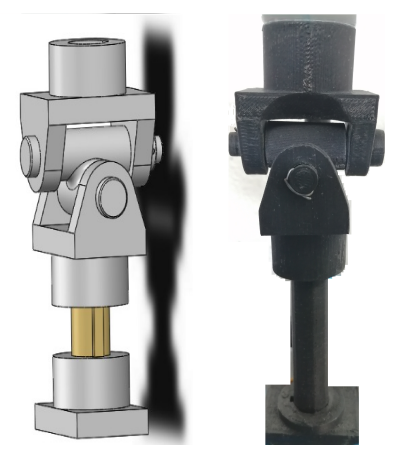

Figura 2: Mecanismo de giro central (pivote). 
Para variar las posiciones angulares $\theta_{X}$ y $\theta_{Y}$, se emplean dos servomotores y se diseña un sistemas de bielas para la transmisión de movimiento de los servomotores a la pantalla táctil. Este sistema consta de dos mecanismos de giro lateral, Figura 3 y dos mecanismo de eslabones con soportes para fijar los servomotores a la base inferior, Figura 4. Los eslabones metálicos que interconectan el servomotor con la barra del mecanismo de giro mostrado en la Figura 4, no fueron construidos por los autores, sino adquiridas por su fabricante.

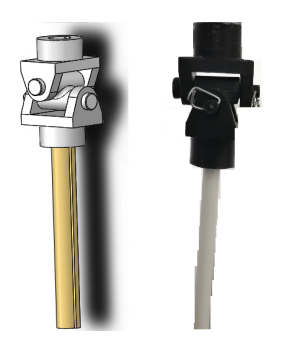

Figura 3: Mecanismo de giro lateral (bielas).

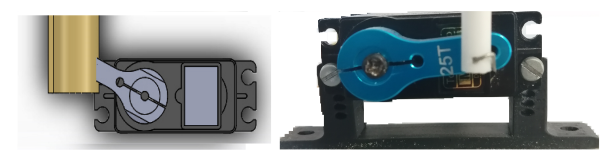

Figura 4: Mecanismo de eslabones con soportes para el servomotor.

La integración de los componentes descritos anteriormente para la construcción de la plataforma bola-plano son mostrados en la Figura 5.

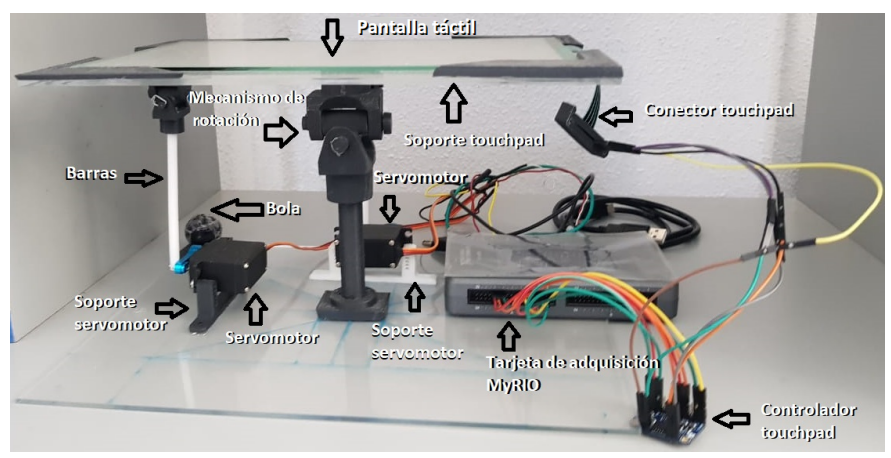

Figura 5: Componentes que conforman la plataforma bola-plano.

Las especificaciones de los componentes tales como: servomotores, pantalla táctil y controlador, se describen en la sección siguiente.

\subsection{Especificaciones de los componentes}

Los servomotores MG996R utilizados para actuar cada eje de giro cuentan con las siguientes características principales siguientes

1. Torque: $9.4 \mathrm{kgf.cm}(4.8 \mathrm{~V})$ y $11 \mathrm{kgf.cm}(6 \mathrm{~V})$.

2. Velocidad de operación: $0.17 \mathrm{~s} / 60^{\circ}(4.8 \mathrm{~V})$ y $0.14 \mathrm{~s} / 60^{\circ}$ $(6 \mathrm{~V})$.
3. Tensión de operación: 4.8 - 7.2 V.

Las características mencionadas anteriormente, se seleccionaron para que dichos servomotores cumplan con las especificaciones de velocidad requeridas.

La pantalla táctil resistiva FTAS00-6.5AS-4 seleccionada presenta las características que siguen:

1. Alimentación: 1mA @ 5V DC.

2. Valor de resistencia X,Y: 250-850, 120-1500 $\Omega$.

3. Fuerza de activación: $1.47 \mathrm{~N}$.

4. Tiempo de respuesta: $10 \mathrm{~ms}$.

5. Dimensión: 6.5”.

6. Área de contacto: $132 \mathrm{~mm} \times 99 \mathrm{~mm}$.

Las principales características que se tomaron en cuenta para la selección de este modelo de pantalla táctil fueron, el tiempo de respuesta y la fuerza necesaria para su activación, dado los requerimientos temporales de la respuesta y el peso de la bola.

El controlador STMPE610 mostrado en la Figura 6, fue utilizado para obtener la lectura de la posición de la bola en la pantalla táctil y luego ser enviado a la tarjeta de adquisición de datos MyRIO-1900, las principales especificaciones se resumen como sigue

1. Tensión de operación: $1.8-3.3 \mathrm{~V}$.

2. Controlador integrado para una pantalla táctil de cuatro hilos.

3. Interfás de comunicación SPI (Serial Peripheral Interface) e $I^{2} C$.

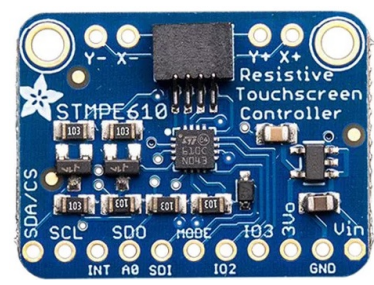

Figura 6: Controlador STMPE610.

Se aprovechó la característica de este controlador de propósito general de contar con un módulo programable para la lectura de pantallas táctil resistivas de cuatro hilos. Además, se utilizó el protocolo de comunicación SPI para obtener los datos en la MyRIO-1900.

La conexión entre el controlador STMPE610 y la tarjeta de adquisición de datos MyRIO-1900 se puede observar en la Figura 7.

En la sección siguiente se describe algunos detalle para obtener la posición de la bola sobre el plano.

\subsubsection{Obtención de la posición de la bola}

Para determinar la posición de la bola sobre el plano se necesita un sensor con buena resolución, exactitud y tiempo de respuesta, ya que la posición cambiará tan rápido como sea la 


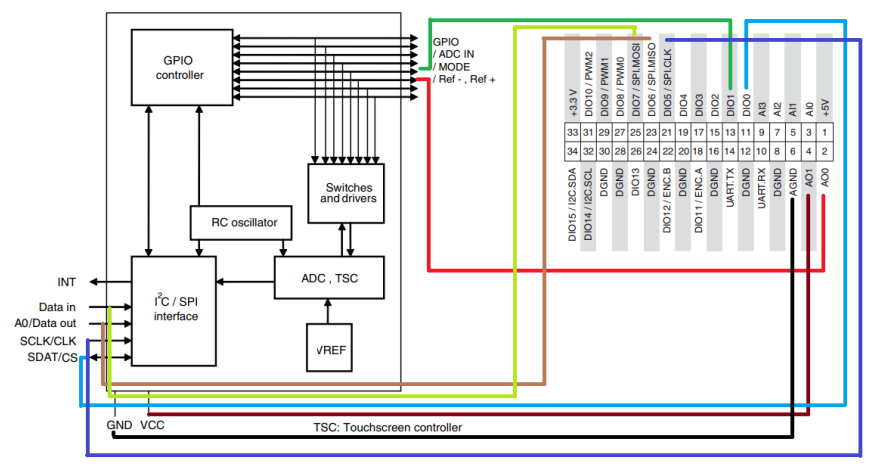

Figura 7: Conexión entre el controlador STMPE610 y la tarjeta de adquisición de datos MyRIO-1900.

aceleración de la bola, por lo tanto se seleccionó como sensor de posición una pantalla táctil resistiva de 4 hilos, la cual cuenta con una respuesta lineal con un error $\pm 1.5 \%$, necesita fuerza de activación de $1 \mathrm{~N}$ (Newton) a $1.47 \mathrm{~N}$ y un tiempo de respuesta menor a $10 \mathrm{~ms}$, estas características hacen que el sensor sea rápido y exacto a la hora de enviar los datos.

Para la adquisición de datos se optó por una tarjeta modelo NI MyRIO-1900, que con ayuda del controlador STMPE610 recibe los datos enviados por la pantalla táctil y por medio del software LabVIEW se procesan los mismos. Para observar la configuración de la comunicación entre el controlador y la tarjeta véase el apéndice A.

A partir del modelo matemático obtenido que representa al bola-plano se procede al diseño y sintonización de una ley de control para cada eje de la plataforma.

\section{Ley de control y sintonización de sus ganancias}

Para la manipulación de la posición de la bola respecto a la variación del plano, se propone un controlador conocido como realimentación de estado, equivalente a un control con acciones proporcional y derivativa (PD), cuya forma es la siguiente:

$$
u=-k x=-k_{1} x_{1}-k_{2} x_{2} ; \quad k=\left[\begin{array}{ll}
k_{1} & k_{2}
\end{array}\right] \in \mathbb{R}^{1 \times 2} .
$$

Así, el polinomio característico del sistema (10) en lazo cerrado con la ley de control (11) es

$$
\begin{aligned}
P(s) & =\operatorname{det}\{(s I-(A-B k))\} \\
& =\operatorname{det}\left\{\left[\begin{array}{cc}
s & -1 \\
\frac{5}{7} g k_{1} & \frac{5}{7} s+g k_{2}
\end{array}\right]\right\} \\
& =s^{2}+\frac{5}{7} g k_{2} s+\frac{5}{7} g k_{1} .
\end{aligned}
$$

Dado que se desea que el polinomio característico (12) sea Hurtwitz, es decir, que la parte real de los valores propios sean negativas, se emplea el método de asignación de polos. El cual consiste en proponer un polinomio deseado cuyas raíces son propuestas por el sintonizador o usuario, mediante la elección adecuada de los valores de $k$.

Debido a que el sistema necesita converger de la forma más rápida al punto de deseado, se propone un polinomio deseado de segundo orden con raíces enteras negativas de la forma

$$
P_{d}(s)=s^{2}+2 a s+a^{2}, a \in \mathbb{Z} .
$$

Igualando los coeficientes del polinomio característico (12) con los coeficientes del polinomio deseado (13) se tiene lo siguiente

$$
\frac{5}{7} g k_{2}=2 a \quad \text { y } \quad \frac{5}{7} g k_{1}=a^{2} .
$$

Despejando las ganancias $k_{2}$ y $k_{1}$, se obtiene que

$$
k_{2}=\frac{14 a}{5 g} \quad \text { y } \quad k_{1}=\frac{7 a^{2}}{5 g} \text {. }
$$

Con cualquier valor de $a>0$ se puede garantizar la estabilidad del sistema, ahora el problema es encontrar un valor para la variable $a$ que evite que el sistema tenga error en estado estacionario, por lo que se aplica el teorema del valor final, el cual nos ayuda a determinar el comportamiento en estado estacionario de un sistema estable. Así, el error en estado estacionario $\left(e_{s s}\right)$ es

$$
e_{s s}=\lim _{t \rightarrow \infty} e(t)=\lim _{s \rightarrow 0} s E(s),
$$

donde $e(t)=r(t)-y(t)$ es el error, $r(t)$ es la referencia y $y(t)$ es la salida del sistema. Por otro lado, considerando una función de transferencia de la forma

$$
\frac{Y(s)}{R(s)}=\frac{1}{s^{2}+2 a s+a^{2}}
$$

y suponiendo que $R(s)=\frac{1}{s}$ es un escalón unitario se tiene que

$$
Y(s)=\frac{1}{s^{2}+2 a s+a^{2}} \frac{1}{s} .
$$

Por lo que

$$
\begin{aligned}
s E(s) & =s\left[\frac{1}{s}-\frac{1}{s^{2}+2 a s+a^{2}} \cdot \frac{1}{s}\right] \\
& =1\left(1-\frac{1}{s^{2}+2 a s+a^{2}}\right) .
\end{aligned}
$$

Aplicando el teorema de valor final

$$
\lim _{s \rightarrow 0} s E(s)=1\left(1-\frac{1}{a^{2}}\right) \longrightarrow 0 \quad \text { si y sólo si } a=1 .
$$

Así, es claro que las ganancias $k_{2}$ y $k_{1}$ son

$$
k_{1}=0.142857 \quad \text { y } \quad k_{2}=0.285714 \text {. }
$$

Con la ley de control ya diseñada, se procede a implementarla en el software LabVIEW, en la sección siguiente se muestran los resultados obtenidos. 


\section{Resultados experimentales}

A continuación, se describe la prueba experimental realizada, además, a partir del experimento se muestran los resultados obtenidos.

\subsection{Prueba experimental}

La prueba experimental consiste en la estabilización de la bola en las coordenadas $X=0.07 \mathrm{~m}$ e $Y=0.117 \mathrm{~m}$, aproximadamente el centro de la pantalla táctil. En este caso, los valores ajustados para las ganancias de los controles son $k_{1}=0.151 \mathrm{y}$ $k_{2}=0.273$, los cuales no varían considerablemente con respecto a los valores obtenidos en la sintonización por el método de asignación de polos.

Por otra parte, las señales de control para los ejes $X$ e $Y$ no se aplican directamente a los servomotores, estas son convertidas a microsegundos $(\mu s)$ en estado alto necesarios para generar la modulación de ancho de pulso. Cabe mencionar que con un valor de $1500 \mu s$ los ejes de los servomotores se encuentran en una posición horizontal, es decir a 90 grados. Con un valor de $1000 \mu s$ el eje se coloca en 110 grados, mientras que con un valor de $2000 \mu s$ el eje del servomotor se coloca en 60 grados.

\subsection{Resultados obtenidos}

A partir de la plataforma diseñada y construida, e implementando la ley de control sintonizada, se prosigue a obtener las gráficas de las señales de error, control y respuesta del sistema a partir de los resultados experimentales realizados para el punto de equilibrio.

La respuesta del sistema en lazo cerrado con el controlador PD para tareas de estabilización es ilustrada en la Figura 8 para el eje X y en la Figura 9 para el eje Y.

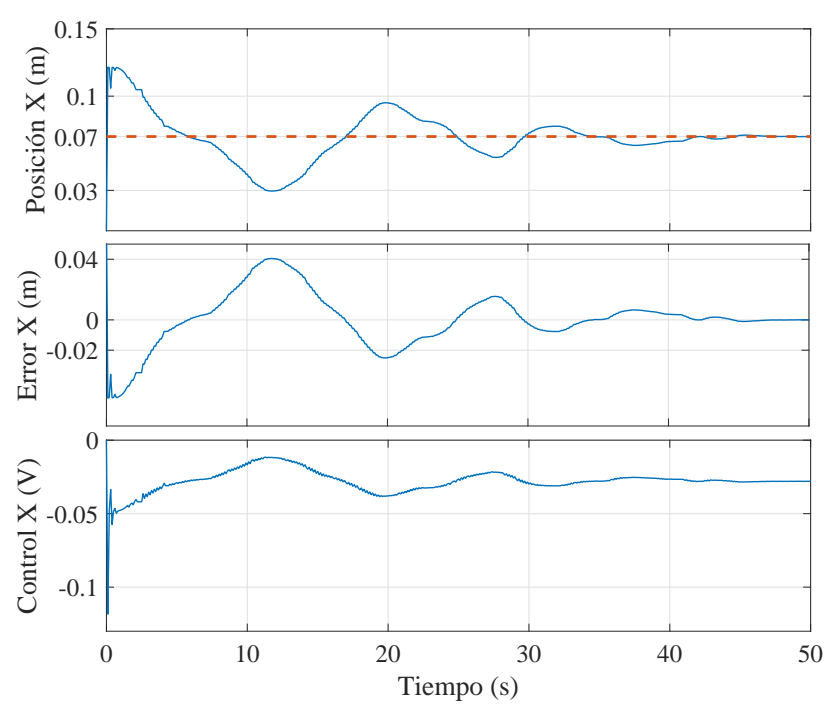

Figura 8: Posición de la bola, señal de error y control para el eje X.

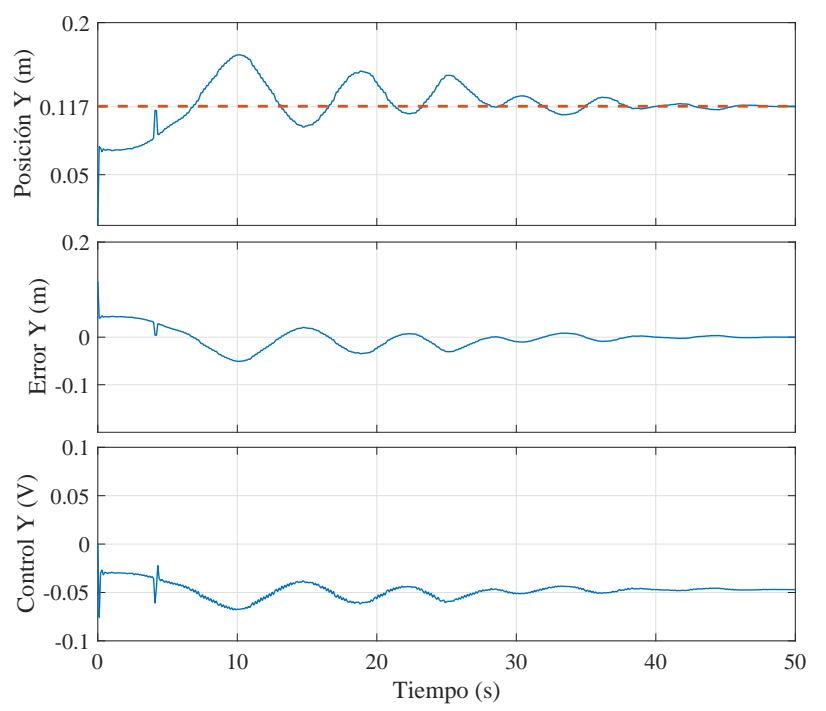

Figura 9: Posición de la bola, señal de error y control para el eje Y.

En las Figuras 8 y 9 los errores son muy cercanos a cero, pero nunca serán exactamente cero, ya que debido a la resolución de la pantalla, el contacto de la bola sobre ésta es siempre sobre varios píxeles a la vez, es decir, aunque la bola esté fija, en la medición de la posición se detectan varios puntos correspondientes a los píxeles que se encuentran alrededor de la posición deseada.

En la siguiente sección se arrojan las conclusiones obtenidas a partir de las pruebas experimentales realizadas.

\section{Conclusiones}

Se ha diseñado y construido una plataforma experimental conocida como bola-plano, con un costo considerablemente inferior al de prototipos comerciales. Mediante la implementación de la ley de control PD en la plataforma desarrollada se han obtenido resultados experimentales esperados. Para la sintonización del controlador se ha empleado el método de asignación de polos, con lo cual se ha logrado estabilizar la plataforma bola-plano de manera sencilla.

La construcción de la estructura se realizó en su mayoría mediante impresiones 3D, logrando mayor resistencia en los mecanismos de rotación y disminuyendo el costo total de la estructura.

Para la intercomunicación entre el controlador STMPE610 y la tarjeta de adquisición de datos MyRIO-1900 se utiliza el protocolo de comunicación SPI, por lo cual se tuvo que realizar desde el inicio toda la configuración del controlador mediante el software LabVIEW, dado que el fabricante del mismo sólo proporciona librerías para ser utilizadas en Arduino o Raspberry.

De los diversos mecanismos para determinar la posición de la bola en la plataforma se pueden encontrar: mediante sistemas de visión, sistemas GPS y mediante pantallas táctiles resistivas o capacitivas. Para el presente proyecto se seleccionó la pantalla 
resistiva dado que es el método más sencillo de implementar, de bajo costo computacional y económico, además de ofrecer los beneficios deseados en cuanto a velocidad de respuesta y precisión.

Con la construcción de la plataforma se cuenta con un prototipo eficiente para ser utilizado por estudiantes en donde se les permita implementar diferentes algoritmos y/o leyes de control.

\section{English Summary}

\section{form.}

Design, construction and control of a Ball-Plane plat-

\section{Abstract}

In this document the design, construction and control of a ball-plane platform is presented. All the components of the platform were designed with CAD software SolidWork and printed with a 3D printer. Also, to stabilize the ball on the plane a Proportional-Derivative (PD) control law is tuned using a mathematical model of the system. The control law is implemented using LabVIEW software and MyRIO-1900 data acquisition card. Finally, the experimental results are obtained.

\section{Keywords:}

ball-plane platform, Computer-Aided Design, PD controller, stabilization of the systems.

\section{Agradecimientos}

Agradecemos al Consejo Nacional de Ciencia y Tecnología (CONACYT) por el apoyo económico brindado mediante la beca de posgrado que nos otorga. Además, al núcleo académico del Instituto de Ciencias Básicas e Ingenierías (ICBI) de la Universidad Autónoma del Estado de Hidalgo (UAEH), por aportarnos sus conocimientos en las diferentes asignaturas que hicieron posible este artículo. A todas las personas que de una manera $\mathrm{u}$ otro aportaron en la realización de todo el proyecto. A todos, muchas gracias.

\section{Referencias}

Awtar, S., Bernard, C., Boklund, N., Master, A., Ueda, D., Craig, K., 2002. Mechatronic design of a ball-on-plate balancing system. Mechatronics 12 (2), 217-228.

B. A. López, N. C. Castillo, L. A. C. L. V. R. V., 2018. Diseño, construcción y estabilización de un sistema bola-balancín. Publicación Semetral Padi (10), 56-61.

Benbrahim, H., Doleac, J., Franklin, J. A., Selfridge, O. G., 1992. Real-time learning: A ball on a beam. In: [Proceedings 1992] IJCNN International Joint Conference on Neural Networks. Vol. 1. IEEE, pp. 98-103.

Colmenares, S. G., Moreno-Armendáriz, M. A., Yu, W., Rodriguez, F. O., 2012. Modeling and nonlinear pd regulation for ball and plate system. In: World Automation Congress 2012. IEEE, pp. 1-6.

Dong, X., Zhao, Y., Xu, Y., Zhang, Z., Shi, P., 2011. Design of pso fuzzy neural network control for ball and plate system. International Journal of Innovative Computing, Information and Control 7 (12), 7091-7103.
Garzón Melo, Y., Garzón Mancera, O. L., et al., 2016. Diseño e implementación de una plataforma bola y plato, para la aplicación de técnicas de control PID y LQR.

Hauser, J., Sastry, S., Kokotovic, P., 1992. Nonlinear control via approximate input-output linearization: The ball and beam example. IEEE transactions on automatic control 37 (3), 392-398.

Keshmiri, M., Jahromi, A. F., Mohebbi, A., Hadi Amoozgar, M., Xie, W.-F., 2012. Modeling and control of ball and beam system using model based and non-model based control approaches. International Journal on Smart Sensing \& Intelligent Systems 5 (1).

Liu, H., Liang, Y., 2010. Trajectory tracking sliding mode control of ball and plate system. In: Informatics in Control, Automation and Robotics (CAR), 2010 2nd International Asia Conference on. Vol. 3. IEEE, pp. 142-145.

Mancera, O. L. G., 2016. Diseño e implementación de una plataforma bola y plato, para la aplicación de técnicas de control PID y LQR. B.S. thesis, Universidad Distrital Francisco José De Caldas.

Mochizuki, S., Ichihara, H., 2013. Generalized Kalman-Yakubovich-Popov lemma based I+PD controller design for ball and plate system. Journal of Applied Mathematics 2013, 1-9.

Tack, H. H., Choo, Y. G., Kim, C. G., Jung, M. W., 1999. The stabilization control of ball-beam using self-recurrent neural networks. In: 1999 Third International Conference on Knowledge-Based Intelligent Information Engineering Systems. Proceedings (Cat. No. 99TH8410). IEEE, pp. 222-225.

Wang, W., Wang, F., Jin, Z., Dowson, D., Hu, Y., 2009. Numerical lubrication simulation of metal-on-metal artificial hip joint replacements: ball-in-socket model and ball-on-plane model. Proceedings of the Institution of Mechanical Engineers, Part J: Journal of Engineering Tribology 223 (7), 1073-1082.

\section{Apéndice A. Diagrama de Flujo}

A continuación se muestra el diagrama de flujo del código implementado en LabVIEW.

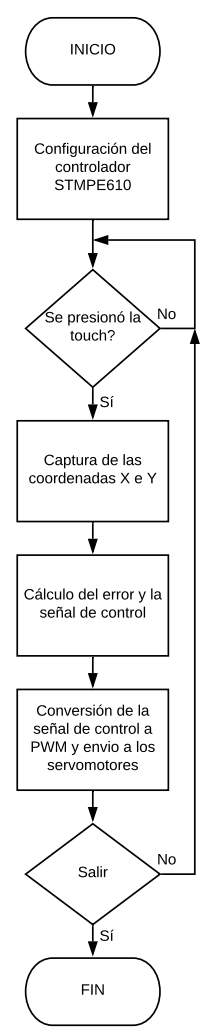

Figura A.10: Diagrama de Flujo. 


\section{Apéndice B. Código implementado en LabVIEW}

A continuación se muestra el código implementado en LabVIEW para la puesta en marcha de la plataforma bola-plano. La Figura B.11 muestra la configuración del controlador STMPE610. Dicho controlador utiliza el protocolo de comunicación SPI con la tarjeta de adquisición de datos MyRIO-1900. En la Figura B.12 se observa el proceso de detección de presión sobre la pantalla táctil, por lo cual se accede al registro del controlador donde se almacena cuándo se presiona la pantalla táctil. Una vez detectada interacción con la pantalla táctil se procede a capturar los registros donde se almacena la posición en las coordenadas $X$ e $Y$; como se puede observar en la Figura B.13, luego se realizan algunas operaciones con dichos registros con el objetivo de organizar y formar los datos exactos de la posición. Con los valores de la posición obtenidos se realiza el cálculo del error y de la señal de control, la cual se aplica a los respectivos servomotores.

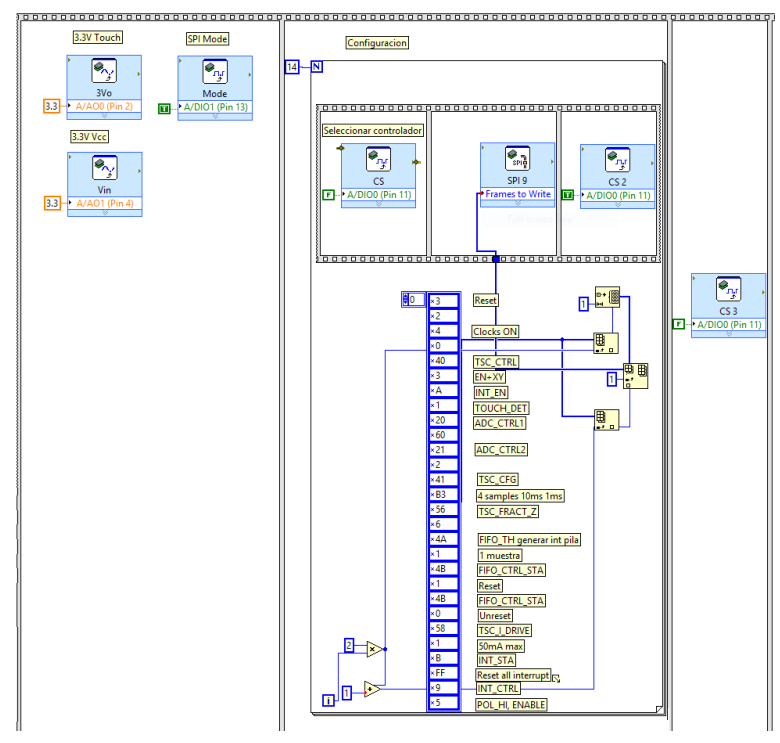

Figura B.11: Configuración del controlador STMPE610.

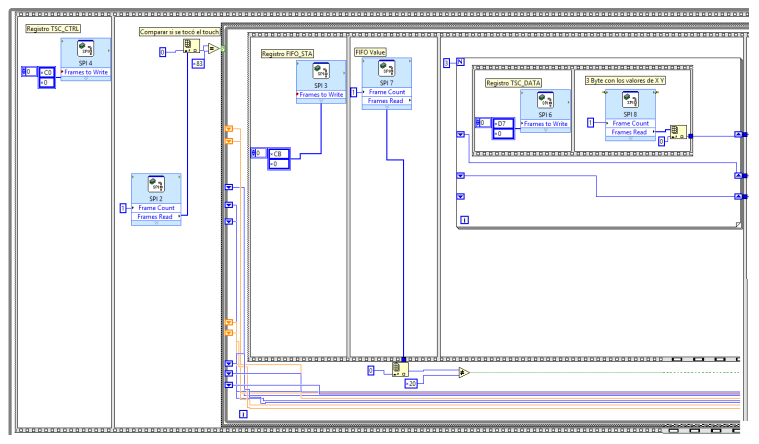

Figura B.12: Detección de la presión sobre la pantalla táctil.

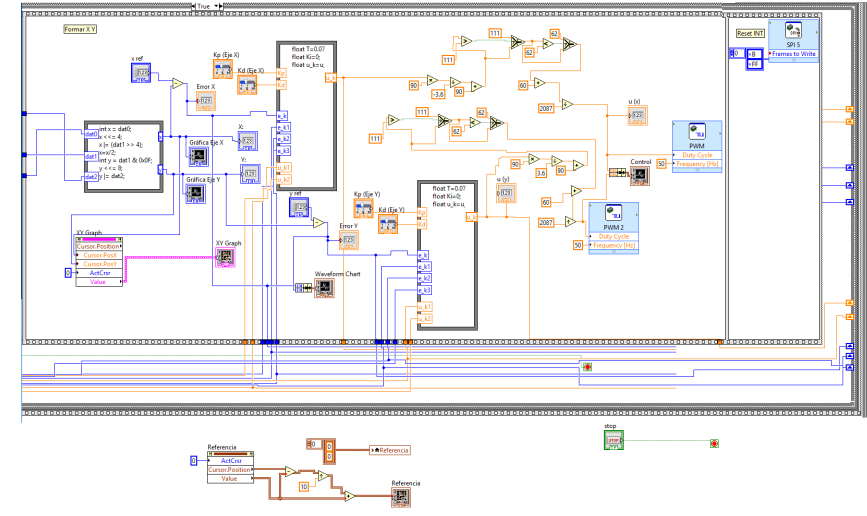

Figura B.13: Obtención de la posición y cálculo de la señal de control.

\section{Apéndice C. Interfaz desarrollada en LabVIEW}

La Figura C.14 muestra la interfaz desarrollada en LabVIEW para la interacción entre el usuario y la plataforma.

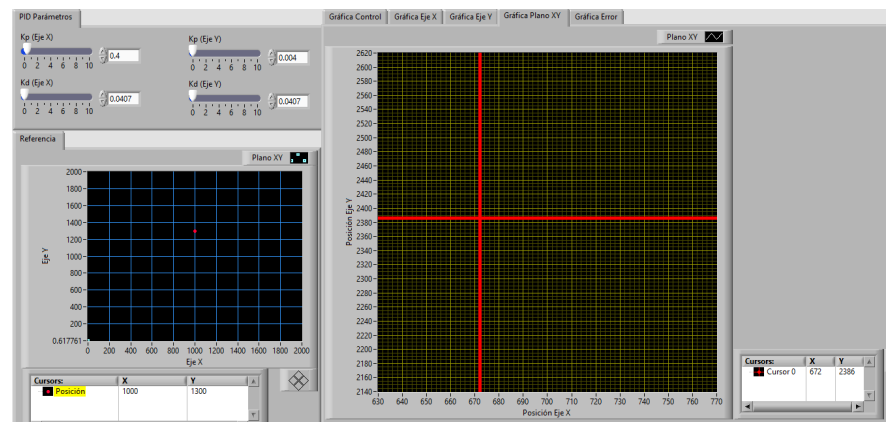

Figura C.14: Interfaz desarrollada en LabVIEW. 